\title{
Plattformökonomie: neue Wettbewerbsregeln - Renaissance der Missbrauchsaufsicht
}

\author{
Die Plattformökonomie entwickelt sich rasant und intensiviert den Wettbewerb. Dieser \\ an sich positiven Entwicklung steht ein hohes Risiko der Marktabschottung durch \\ marktbeherrschende Plattformen gegenüber. Einige Plattformunternehmen dominieren die \\ Weltmärkte und verfügen über eine erhebliche Machtstellung, die eine Nachjustierung des \\ Kartellrechts erforderlich macht. Dies ist jetzt im Rahmen der 10. Novelle des Gesetzes gegen \\ Wettbewerbsbeschränkungen (GWB) geplant. Richtigerweise wird der Fokus hier auf die \\ kartellrechtliche Missbrauchsaufsicht und weniger auf die Fusionskontrolle gelegt.
}

\begin{abstract}
Das dramatische Wachstum und die inzwischen sehr starke Marktstellung und enorme Marktkapitalisierung einiger Unternehmen der Plattformökonomie werden von vielen Beobachtern mit Skepsis und Sorge betrachtet. In der westlichen Welt dominieren vor allem Google, Amazon, Facebook, Apple und Microsoft (GAFAM) ganze Branchen und gehören längst zu den wertvollsten Unternehmen der Welt. Eine ähnliche Rolle spielen Tencent, Alibaba und Samsung in Asien. Die Sorge um die Macht dieser Unternehmen treibt Wettbewerbsbehörden, Juristen, Ökonomen und Sozialwissenschaftler sowie politische Entscheidungsträger zunehmend um. Diverse Untersuchungen von Wettbewerbsbehörden ${ }^{1}$ sowie weitere Studien ${ }^{2}$ spiegeln diese wachsende Sorge wider. In Deutschland hat zuletzt
\end{abstract}

(c) Der/die Autor(en) 2020. Open Access: Dieser Artikel wird unter der Creative Commons Namensnennung 4.0 International Lizenz (http:// creativecommons.org/licenses/by/4.0/deed.de) veröffentlicht.

Open-Access wird durch die ZBW - Leibniz-Informationszentrum Wirtschaft gefördert.

1 Siehe z.B. Autorité de la concurrence \& Bundeskartellamt: Competition Law and Data, Paris, Bonn 2016; OECD: Rethinking Antitrust Tools for Multi-Sided Platforms, Paris 2018; Australian Competition and Consumer Commission (ACCC): Digital Platforms Inquiry: Final Report, Canberra 2019.

2 H. Schweitzer, J. Haucap, W. Kerber, R. Welker: Modernisierung der Missbrauchsaufsicht für marktmächtige Unternehmen, Baden-Baden 2018; J. Crémer, Y.-A. de Montjoye, H. Schweitzer: Competition Policy for the Digital Era: Final Report, Publications Office of the European Union, Luxemburg 2019; J. Furman, D. Coyle, A. Fletcher, D. McAuley, P. Marsden: Unlocking Digital Competition, Report of the Digital Competition Expert Panel, UK Government, London 2019.

Prof. Dr. Justus Haucap ist Direktor des Düsseldorf Institute for Competition Economics (DICE) an der Heinrich-Heine-Universität in Düsseldorf. die Expertenkommission Wettbewerbsrecht 4.0 ihren Abschlussbericht an den Bundeswirtschaftsminister übergeben. ${ }^{3}$ Auslöser der teils disruptiven Entwicklungen auf vielen Märkten sind zwei Faktoren, die Wertschöpfungsketten und Wettbewerbsprozesse in vielen Branchen und Märkten grundlegend verändert haben und weiter verändern werden. Erstens haben digitale Plattformen stark an Bedeutung gewonnen, ${ }^{4}$ da sie entweder traditionelle Intermediationsformen wie etwa im Einzelhandel, im Finanzsektor, in der Medienbranche oder im Dienstleistungsgewerbe (Makler, Reisebüros etc.) zunehmend ersetzen oder indem sie Transaktionen erleichtert haben, die zuvor aufgrund von Koordinationsproblemen und/oder mangelndem Vertrauen nicht stattgefunden haben wie etwa im Bereich der Sharing Economy. ${ }^{5}$ Und zweitens sind Daten zu einem kritischen Input für Produktions- und Vertriebsprozesse in vielen Branchen wie Landwirtschaft, Industrieproduktion, Logistik, Marketing, Einzelhandel, Finanzen und vielen anderen Teilen der Wirtschaft geworden. ${ }^{6}$

3 Expertenkommission Wettbewerbsrecht 4.0: Ein neuer Wettbewerbsrahmen für die Digitalwirtschaft, Berlin 2019.

4 Vgl. z. B. D. S. Evans, R. Schmalensee: Matchmakers: The New Economics of Multisided Platforms, Boston 2016; G. G. Parker, M. W. van Alstyne, S. P. Choudary: Platform Revolution: How Networked Markets Are Transforming the Economy and How to Make Them Work for You, Norton, New York 2016; J. Haucap, U. Heimeshoff: Google, Facebook, Amazon, eBay: Is the Internet Driving Competition or Market Monopolization?, in: International Economics and Economic Policy, 11. Jg. (2014), H. 1-2, S. 49-61.

5 Vgl. etwa J. Dörr, N. Goldschmidt, F. Schorkopf (Hrsg.): Share Economy: Institutionelle Grundlagen und gesellschaftspolitische Rahmenbedingungen, Tübingen 2018; C. Busch et al.: Sharing Economy in Deutschland: Stellenwert und Regulierungsoptionen für Beherbergungsdienstleistungen, Baden-Baden 2019.

6 Vgl. z. B. V. Mayer-Schönberger, K. Cukier: Big Data: A Revolution That Will Transform How We Live, Work, and Think, London 2013; D. L. Rogers: Digital Transformation Playbook: Rethink Your Business for the Digital Age, New York 2016; K. Morik, W. Krämer (Hrsg.): Daten - wem gehören sie, wer speichert sie, wer darf auf sie zugreifen?, Paderborn 2018. 
Weder Plattformen noch die Nutzung von Daten sind dabei völlig neue Phänomene. Plattformen dienten schon immer zur Organisation von Transaktionen. Mittelalterliche Märkte und Messen sind frühe Beispiele für Plattformmärkte, organisierte Börsen für Wertpapiere und Rohstoffe sind weitere Beispiele, ebenso Medien wie Zeitungen oder Free-TV-Sender. Es gibt jedoch zwei wichtige Entwicklungen in Bezug auf Plattformen. Während traditionell Transaktions- und Transport- oder Reisekosten sowie Kapazitätsgrenzen dem Plattformwachstum natürliche Grenzen gesetzt haben, sind diese Kosten jetzt stark gesunken. Der „Death of Distance“ wie die Amerikaner sagen und die gesunkenen Transaktionskosten haben zu einem enormen Plattformwachstum geführt. Ebenso werden Daten von Unternehmen seit jeher für die Konzeption des Produktangebotes und zur Verbesserung von Produktionsprozessen verwendet. Da die Kosten für die Erhebung, Speicherung, Verarbeitung und Analyse von Daten jedoch drastisch gesunken sind, werden immer mehr Daten verwendet. ${ }^{7}$ Diese beiden Entwicklungen, d. h. die zunehmende Bedeutung von Plattformen auf der einen Seite und die Rolle der Daten als kritische Ressource auf der anderen Seite, sind die wesentlichen Treiber des Strukturwandels in der digitalen Wirtschaft.

\section{Plattformökonomie}

In der ökonomischen Literatur wird der Begriff der Plattform oft synonym zu dem des zwei- oder mehrseitigen Marktes (multi-sided oder two-sided market) verwendet. Gemeint ist in aller Regel dasselbe. Von (mehrseitigen) Plattformen wird gesprochen, wenn erstens mindestens zwei unterschiedliche Nutzergruppen (z. B. Käufer und Verkäufer, Fahrer und Fahrgäste, Hotels und Reisende etc.) über eine Plattform vermittelt interagieren, und zweitens die Präsenz der einen Gruppe den Nutzen der anderen Gruppe durch indirekte Netzwerkeffekte positiv oder negativ beeinflusst. $^{8}$

Auch Informationsintermediäre sind an sich keine neuen Erscheinungen der digitalen Ökonomie. Sie haben

7 Vgl. auch A. Agrawal, J. Gans, A. Goldfarb: Prediction Machines: The Simple Economics of Artificial Intelligence, Boston 2018.

8 Vgl. J. Wright: One-sided Logic in Two-sided Markets, in: Review of Network Economics, 3. Jg. (2004), H. 1, S. 42-63; M. Armstrong: Competition in Two-sided Markets, in: RAND Journal of Economics, 37. Jg. (2006), H. 3, S. 668-691; M. Peitz: Marktplätze und indirekte Netzwerkeffekte, in: Perspektiven der Wirtschaftspolitik, 7. Jg. (2006), H. 3, S. 317-333. Teilweise wird auch eine engere Definition verwendet. So sprechen Rochet und Tirole von einem zweiseitigen Markt, wenn die Struktur der Preise, welche die beiden Nutzergruppen zu entrichten haben, das Transaktionsvolumen insgesamt beeinflusst. Vgl. J.-C. Rochet, J. Tirole: Platform Competition in Two-sided Markets, in: Journal of the European Economics Association, 1. Jg. (2003), H. 4, S. 990-1029; J.-C. Rochet, J. Tirole: Two-sided Markets: A Progress Report, in: RAND Journal of Economics, 37. Jg. (2006), H. 3, S. 645-667. auf manchen Märkten - etwa Börsen auf Kapital- und Rohstoffmärkten, Immobilienmakler oder Anlageberater - schon immer eine Rolle gespielt (und sich teilweise als regelungsbedürftig erwiesen). Auch Reisebüros, Versicherungsmakler oder Zimmervermittlungen waren schon in der Vergangenheit Informationsintermediäre mit Plattformcharakter. Neu ist allerdings die nahezu durchgängige Bedeutung von digitalen Plattformen als Vermittler im Internet in unzähligen Branchen. Neu ist ferner die Prominenz, die dabei das Geschäftsmodell der mehrseitigen Plattform erlangt hat.

Technische Voraussetzung für die große praktische Bedeutung digitaler Plattformen als neue Intermediäre und Marktplätze war die rasante Entwicklung der Datenspeicherungs- und Datenverarbeitungsmöglichkeiten, die nicht nur die systematische Sammlung und Verarbeitung der verfügbaren Informationen auf Anbieterseite möglich gemacht hat, sondern darüber hinaus die systematische automatisierte Speicherung und Auswertung von Informationen über das Nutzerverhalten. Das effiziente „Matching“ der (Informations-)Angebote entsprechend den Präferenzen ist zum Kern vieler digitaler Plattformen geworden.

Für den Erfolg von mehrseitigen Plattformen ist zudem ganz generell das Ausschöpfen indirekter und teils auch direkter Netzwerkeffekte mitentscheidend. Folgt der Nutzen einer Plattform gerade aus der tatsächlichen oder auch potenziellen Interaktion zwischen den Marktseiten, so ist entscheidend, dass alle relevanten Marktseiten in hinreichender Zahl partizipieren, um die Plattform für die jeweils anderen Marktseiten attraktiv zu machen. In vielfältiger Weise befördern Plattformen so die private, soziale und wirtschaftliche Interaktion.

In ökonomischer Hinsicht erhöhen Plattformen so zunächst einmal die Angebotsvielfalt. Diejenigen Plattformen, die auf ein informationelles (Informationsplattformen) oder transaktionsbezogenes (Transaktionsplattformen) „Matchmaking" ausgerichtet sind, haben dabei grundsätzlich starke Anreize, Strukturen zu schaffen, die Transaktionskosten für alle Plattformseiten senken und diese passgenau und präferenzgerecht zusammenführen. ${ }^{9}$ Betrachten wir etwa das Beispiel einer Transaktionsplattform: Ihr Ziel ist es regelmäßig, den Marktplatz derart zu strukturieren, dass die Transaktionskosten minimiert und so das Transaktionsvolumen erhöht wird. Mit dem Transaktionsvolumen steigen auch die Provisionsansprüche der Plattform. Es ist daher grundsätzlich im Eigeninteresse der Plattform, Marktregeln aufzustellen und durchzusetzen, welche die

9 Ausführlich und unter Hinweis auf die Unterschiede zwischen Transaktions- und Informationsplattformen vgl. A. Engert: Digitale Plattformen in: Archiv für die civilistische Praxis, 218. Jg. (2018), H. 2-4, S. 307 ff. 
Informationsasymmetrien in Bezug auf die Qualität der angebotenen Leistungen reduzieren, so das Vertrauen der Nutzerseite in die Zuverlässigkeit und Nutzerfreundlichkeit der Plattform erhöhen und opportunistisches Verhalten der Plattformnutzer minimieren. Bewertungssysteme, die sowohl eine Bewertung von Produkten bzw. Dienstleistungen als auch die Bewertung von Händlern ermöglichen, dienen dazu, das Vertrauen in die über die Plattform vermittelten Transaktionen zu steigern. ${ }^{10}$ Digitale Informations- und Transaktionsplattformen haben damit im Ausgangspunkt Anreize, als neutrale und „ehrliche Makler“ zu agieren, und dementsprechend pro-kompetitive Wirkungen.

Gleichwohl können Anreize für missbräuchliche Strategien entstehen, die den Wettbewerb ausbremsen und Ineffizienzen erzeugen. Plattformen können erstens ein Interesse daran haben, Wettbewerb anderer Plattformen abzuwehren - gegebenenfalls auch mithilfe wettbewerbswidriger Abschottungsstrategien. Zweitens können Anreize bestehen, eine auf einem Markt bestehende Marktmacht auf angrenzende Märkte zu übertragen. Eine etablierte Fallgruppe in diesem Bereich betrifft Kopplungsstrategien. ${ }^{11}$ Als missbrauchsanfällig gelten zudem insbesondere vertikal integrierte Plattformen, die einerseits als Organisator eines Marktplatzes auftreten, andererseits aber zugleich selbst als Anbieter auf diesem Marktplatz tätig sind, wie dies etwa bei Amazon Marketplace der Fall ist. Verfügt eine solche Plattform als Organisator des Marktplatzes über eine marktbeherrschende Stellung auf dem „Markt für Marktplätze“, sodass sie in ihrem Verhalten durch den Wettbewerb nicht mehr hinreichend diszipliniert ist, so kann sie über Möglichkeiten und auch Anreize verfügen, Informationsvorteile, Ressourcen (z.B. Daten) und Lenkungsmöglichkeiten, über die sie als Plattform verfügt, zur Ausdehnung ihrer Machtposition auf angrenzende Märkte auszunutzen. Beispiele hierfür können die bevorzugte Anzeige eigener Angebote im Ranking ${ }^{12}$ oder die Nutzung

10 Vgl. G. Bolton, B. Greiner, A. Ockenfels: Engineering Trust: Reciprocity in the Production of Reputation Information, in: Management Science, 59. Jg. (2013), H. 2, S. 265-285.; L. Einav, C. Farronato, J. Levin: Peer-to-Peer Markets, in: Annual Review of Economics, 8. Jg. (2016), H. 1, S. 615-635; S. Tadelis: Reputation and Feedback Systems in Online Platform Markets, in: Annual Review of Economics, 8. Jg. (2016), H. 1, S. 321-340; A. Engert, a. a. O., S. 349 ff.

11 Europäische Kommission, Entscheidung vom 18.7.2018, Case AT.40099, zu Google Android, noch nicht veröffentlicht, März 2020. Vgl. Europäische Kommission: Kartellrecht: Kommission verhängt Geldbuße von 4.34 Milliarden Euro gegen Google wegen illegaler Praktiken bei Android-Mobilgeräten zur Stärkung der beherrschenden Stellung der Google-Suchmaschine, Pressemitteilung vom 18.7.2018, https://ec.europa.eu/commission/presscorner/detail/de/ IP_18_4581 (20.3.2020).

12 Vgl. z.B. Europäische Kommission, Entscheidung vom 27.6.2017, Case AT.39740, bekannt gegeben unter Az. C(2017) 4444, zu Google Shopping; auch Monopolkommission: Wettbewerbspolitik: Herausforderung digitale Märkte, Sondergutachten 68, Baden-Baden 2015, Rn. 391-395. des umfassenden Zugriffs auf Daten der auf der Plattform tätigen Anbieter sein, um dem eigenen (konzerninternen) Angebot in besonders gewinnträchtigen Marktsegmenten Vorteile zu verschaffen. Im Zentrum dieser Diskussion stehen daher mögliche Verhaltenspflichten vertikal integrierter Plattformen. ${ }^{13}$ Bei Transaktionsplattformen kann zudem der Zugriff der Plattform auf Daten insbesondere solcher Anbieter problematisch sein, die zur eigenen Retail-Tochter im Wettbewerb stehen.

Allerdings sind die Anreize, Konkurrenzangebote zu benachteiligen, auch in dem Umfang beschränkt, in dem solche Praktiken die Attraktivität der Plattform als Ganzes für die Plattformnutzer beeinträchtigen - bei Business-toConsumer-Plattformen insbesondere die Attraktivität für die Verbraucher. Nicht immer muss eine Plattform infolge einer Selbstbevorzugung aber mit einer erheblichen Abwanderung von Verbrauchern rechnen, zumal wenn die Selbstbevorzugung ein nur sporadisch auftretendes Verhalten und/oder für Nutzer schwer zu erkennen ist.

\section{Wettbewerb zwischen Plattformen}

Die Intensität des Wettbewerbs in Plattformmärkten wird maßgeblich einerseits durch direkte und indirekte Netzwerkeffekte in Kombination mit Größenvorteilen (economies of scale) bestimmt, andererseits durch die Möglichkeiten, auf mehreren Plattformen parallel aktiv zu sein, also Multi-Homing zu betreiben. ${ }^{14}$ Als Folge der Kombination aus indirekten Netzwerkeffekten und Skalenvorteilen können Plattformmärkte oft stärker konzentriert sein als traditionelle Märkte..$^{15}$ Allerdings ist keineswegs jeder Markt für digitale Plattformen automatisch hoch konzentriert. Gegenbeispiele sind Online-Reisevermittler, Buchungsplattformen für Ferienwohnungen und viele Online-Dating-Websites, auf denen mehrere konkurrierende Plattformen (noch) nebeneinander existieren. Die Existenz indirekter Netzeffekte reicht selbst in Kombination mit Skalenvorteilen nicht automatisch aus, um eine hohe Marktkonzentration oder gar ein Monopol entstehen zu lassen.

Ist Multi-Homing möglich, so können auch bei einem Wettbewerb zwischen verschiedenen Plattformen alle Netzwerkeffekte vollständig realisiert werden. Theoretisch könnten sich alle Nutzer beider Marktseiten auf einer, zwei oder mehreren Plattformen registrieren und so den Wett-

13 Vgl. insbesondere J. Furman, D. Coyle, A. Fletcher, D. McAuley, P. Marsden, a.a.O.

14 Vgl. z.B. D. S. Evans, R. Schmalensee: The Antitrust Analysis of Multi-sided Platform Businesses, in: R. Blair, D. Sokol (Hrsg.): Oxford Handbook on International Antitrust Economics, 1. Jg. (2015), Oxford, S. 404-449; J. Haucap, T. Stühmeier: Competition and Antitrust in Internet Markets, in: J. Bauer, M. Latzer (Hrsg.): Handbook on the Economics of the Internet, Cheltenham 2016, S. 183-210.

15 J. Haucap, U. Heimeshoff, a.a. O. 
bewerb zwischen den Plattformen befördern, ohne auf die Netzwerkeffekte zu verzichten. Der oft geschilderte Zielkonflikt zwischen der Realisierung von Netzwerkeffekten einerseits und dem Bestehen von Wettbewerb andererseits besteht nicht zwingend, wenn das Multi-Homing auf beiden Marktseiten hinreichend einfach ist und die Netzwerkeffekte aus der Vielzahl der möglichen Optionen für Transaktionen resultieren, aber nicht unbedingt daraus, mit möglichst vielen Nutzern auch tatsächlich Transaktionen abzuwickeln.

Allerdings sind auch die Anreize zur Marktabschottung für Online-Plattformen in der Regel stärker als auf traditionellen Märkten, da Plattformen auf „kippligen Märkten“ oder sogenannten „winner takes all“-Märkten operieren. Segal und Winston haben bereits gezeigt, dass es bei hinreichenden Skalenvorteilen schon ausreichen kann, kleine Marktanteile zu behaupten, um einen Markt effektiv zu verschlieBen. ${ }^{16}$ Der Grund ist, dass selbst effiziente Wettbewerber möglicherweise nicht in der Lage sind, eine mindestoptimale Größe zu erreichen, wenn hinreichend viele Kunden Wechselkosten haben oder loyal oder träge sind.

Diese Logik wird noch verstärkt, wenn es zusätzlich zu Skalenvorteilen Netzwerkeffekte gibt - eine für die meisten digitalen Plattformen eher charakteristische Situation. Denn Plattformmärkte sind aufgrund der Netzeffekte tendenziell leichter zu monopolisieren. Nicht umsonst verfolgen viele Plattformen daher eine aggressive Wachstumsstrategie, um schnell eine kritische Masse an Nutzern zu erreichen. Darüber hinaus ist der Markteintritt in einen konzentrierten Plattformmarkt aufgrund des „Henne-und-Ei-Problems“"17 deutlich schwieriger als auf traditionellen Märkten. Reichte es traditionell, eine Kundengruppe, potenzielle Käufer, zu gewinnen, müssen auf Plattformen zugleich zwei Kundenkreise erschlossen werden. Ohne attraktive Angebote durch Anbieter einer Ware oder Dienstleistung bleibt eine Plattform für Käufer uninteressant. Ohne hinreichend viele potenzielle Käufer jedoch ist eine Plattform auch für Verkäufer nicht attraktiv. Die Notwendigkeit, parallel Käufer und Verkäufer zu attrahieren, macht den Markteintritt bei Plattformen relativ schwierig. Somit erschweren Netzwerkeffekte und dieses „Henne-und-Ei-Problem“ den Markteintritt nach einer etwaigen Monopolisierung eines Plattformmarkts. Netzwerk- sowie Skaleneffekte führen daher zu „winner takes all“-Märkten oder „kippligen Märkten“ und damit einer erhöhten Gefahr der strategischen Marktabschottung, vor

16 I. R. Segal, M. D. Whinston: Naked Exclusion: Comment, in: American Economic Review, 90. Jg. (2000), H. 1, S. 296-309.

17 Vgl. B. Caillaud, B. Jullien: Chicken \& Egg: Competition among Intermediation Service Providers, in: RAND Journal of Economics, 34. Jg. (2003), H. 2, S. 309-328. allem wenn es für viele Nutzer schwierig oder unattraktiv ist, sich auf mehreren Plattformen zu bewegen. ${ }^{18}$

Um das Multi-Homing zu unterbinden oder zu erschweren, können marktstarke Plattformen zahlreiche Strategien verfolgen. Eine recht simple Möglichkeit ist der Abschluss von Exklusivverträgen mit hinreichend vielen Teilnehmern mindestens einer Marktseite. Dies kann jedoch bereits heute kartellrechtlich untersagt werden, sofern marktbeherrschende Unternehmen involviert sind. Allerdings können Teilnehmer auch durch Treueprogramme oder Flatrates effektiv vom Multi-Homing abgehalten werden. Zudem gibt es bei Plattformen wie eBay oder Airbnb, wo neben indirekten Netzwerkeffekten auch die Reputation des Nutzers eine hohe Relevanz hat, ${ }^{19}$ nur reduzierte Anreize zum MultiHoming, da die Reputation eines Nutzers und damit auch seine Erlöse von der Zahl der bereits über die Plattform durchgeführten Transaktionen abhängen. Da die Reputation plattformspezifisch ist, hat ein Plattformwechsel hohe Kosten für die Nutzer, da es schwierig, wenn nicht gar unmöglich ist, die eigene Reputation von einer Plattform auf eine andere zu übertragen. ${ }^{20}$ Der Schutz von Möglichkeiten des Multi-Homings ist eine zentrale Aufgabe des Kartellrechts, um wirksamen Wettbewerb auf Plattformmärkten aufrecht zu erhalten.

Allerdings ist auch richtig, dass der Wettbewerb zwischen mehreren Plattformen im Vergleich zu monopolistischen Marktstrukturen nicht immer automatisch effizienzfördernd ist. Während Wettbewerb zwischen mehreren Unternehmen auf traditionellen Märkten fast immer vorteilhaft für Verbraucher ist (sofern der betreffende Markt nicht durch natürliche Monopolbedingungen oder gravierende Informationsasymmetrien gekennzeichnet ist), gilt diese Daumenregel nicht immer für Plattformmärkte. Selbst wenn die Schaffung mehrerer Plattformen keine Duplikation der Fixkosten bedingt, kann das Nebeneinander mehrerer Plattformen ineffizient sein, wenn dadurch Netzwerkeffekte nicht ausgeschöpft werden. Wie Caillaud/Jullien und Jullien gezeigt haben, kann eine Monopolplattform effizient sein, da Netzwerkeffekte maximiert werden, wenn

18 Vgl. auch M. Katz: Exclusionary Conduct in Multi-sided Markets, in: OECD (Hrsg.), a.a. O.; sowie A. Amelio, L. Karlinger, T. Valletti: Exclusionary Practices and Two-sided Platforms, in: OECD (Hrsg.), a.a. O.; $\mathrm{H}$. Vasconcelos: Is Exclusionary Pricing Anticompetitive in Two-sided Markets?, in: International Journal of Industrial Organization, 40. Jg. (2015), H. C, S. 1-10.

19 Vgl. dazu schon M. I. Melnik, J. Alm: Does a Seller's Ecommerce Reputation Matter? Evidence from eBay Auctions, in: Journal of Industrial Economics, 50. Jg. (2002), H. 3, S. 337-349; P. Bajari, A. Hortaçsu: Economic Insights from Internet Auctions, in: Journal of Economic Literature, 42. Jg. (2004), H. 2, S. 457-486.

20 Vgl. J. Haucap, T. Wenzel: Ist eBay unbestreitbar ein nicht-bestreitbares Monopol? Monopolisierungsgefahren bei Online-Marktplätzen, in: R. Dewenter, J. Kruse (Hrsg.): Wettbewerbsprobleme im Internet, München 2009, S. 7-34. 
es allen gelingt, sich über eine einzige Plattform zu koordinieren. ${ }^{21}$ Starke Netzwerkeffekte können daher leicht zu hoch konzentrierten Marktstrukturen führen, aber starke Netzwerkeffekte können diese hochkonzentrierten Marktstrukturen auch effizient sein lassen, ${ }^{22}$ solange Multi-Homing nicht möglich ist. Ist Multi-Homing jedoch auf beiden Seiten der Plattform prinzipiell möglich und wird dies auch praktiziert, so ist - ohne Gegenbeweis - nicht davon auszugehen, dass monopolistische Marktstrukturen automatisch effizient sind.

\section{Begrenzung von Marktmacht auf Plattformmärkten}

Klassische Instrumente der Marktmachtbegrenzung in einer Marktwirtschaft sind zum einen das Kartellrecht, zum anderen Regulierungsmaßnahmen. Die Grenze zwischen Kartellrecht und Regulierung verläuft dabei zunehmend fließend. ${ }^{23}$ Ganz allgemein gesprochen versucht die Regulierung, bestimmte Praktiken von Unternehmen, oftmals sektorenspezifisch, ex ante unter Genehmigungsvorbehalt zu stellen und gegebenenfalls konkrete Wettbewerbsparameter (wie etwa den Preis für ein Produkt) festzulegen oder zumindest zu begrenzen. Regelmäßige Diskussionen drehen sich daher oftmals um regulatorische Begrifflichkeiten, die den Ausgangspunkt bilden, ob ein Unternehmen, Produkt oder eine Vorgehensweise sich unter einen bestimmten Begriff subsumieren lässt und damit die Regulierung anwendbar ist.

Das Kartellrecht hingegen gilt in den meisten Teilen sektorenübergreifend und betrachtet regelmäßig keine Gruppe von Produkten oder Praktiken (auch wenn es etwa durch die europäischen Gruppenfreistellungsverordnungen Ausnahmen gibt), sondern jeden Einzelfall getrennt. Dabei besteht das Kartellrecht aus drei Gebieten, die allesamt dazu dienen, eine missbräuchliche Ausnutzung von Marktmacht zu unterbinden. Dies ist erstens das sogenannte Kartellverbot, wie es in $\S 1$ GWB und Art. 101 Vertrag über die Arbeitsweise der Europäischen Union (AEUV) angelegt ist, das Vereinbarungen zwischen Unternehmen, Beschlüsse von Unternehmensvereinigungen und aufeinander abgestimmte Verhaltensweisen verbietet, wenn diese eine Ver-

21 B. Caillaud, B. Jullien, a. a. O.; B. Jullien: Two-sided Markets and Electronic Intermediaries, in: G. Illing, M. Peitz (Hrsg.): Industrial Organization and the Digital Economy, Cambridge MA 2006, S. 272-303.

22 Vgl. auch E. G. Weyl: A Price Theory of Multi-sided Platforms, in: American Economic Review, 100. Jg. (2010), H. 4, S. 1642-1672; A. Chandra, A. Collard-Wexler: Mergers in Two-sided Markets: An Application to the Canadian Newspaper Industry, in: Journal of Economics and Management Strategy, 18. Jg. (2009), H. 4, S. 1045-1070.

23 Vgl. J. Haucap, J. Kruse: Ex-Ante-Regulierung oder Ex-Post-Aufsicht für netzgebundene Industrien?, in: Wirtschaft und Wettbewerb, 54. Jg. (2004), H. 3, S. 266-275; J. Haucap, A. Uhde: Regulierung und Wettbewerbsrecht in liberalisierten Netzindustrien aus institutionenökonomischer Perspektive, in: ORDO: Jahrbuch für die Ordnung von Wirtschaft und Gesellschaft, Bd. 59 (2008), S. 237-262. hinderung, Einschränkung oder Verfälschung des Wettbewerbs bezwecken oder bewirken. Zweitens ist der Missbrauch marktbeherrschender Stellungen durch ein oder mehrere Unternehmen in § 19 GWB und Art. 102 AEUV verboten. In Deutschland sind darüber hinaus nach § $20 \mathrm{GWB}$ auch bereits bestimmte Verhaltensweisen von Unternehmen mit relativer oder überlegener Marktmacht untersagt. Dieses Missbrauchsverbot betrifft somit Unternehmen, die nicht unbedingt marktbeherrschend sind, aber doch über eine relative oder überlegene Marktmacht verfügen. Drittens wird in $\S \S 35$ - 43a GWB die Fusionskontrolle geregelt. Auf europäischer Ebene entspricht dem die „Verordnung (EG) Nr. 139/2004 des Rates vom 20. Januar 2004 über die Kontrolle von Unternehmenszusammenschlüssen“.

Zwischen Kartellverbot und Missbrauchsaufsicht einerseits und Fusionskontrolle andererseits besteht dabei durchaus ein institutioneller Zusammenhang. Die in Deutschland erst 1974 und in der EU erst 1989 eingeführte Fusionskontrolle ist - ökonomisch betrachtet - das Eingeständnis, dass das Kartellverbot und die kartellrechtliche Missbrauchsaufsicht nur sehr unvollständig funktionieren. Würden - rein hypothetisch - Kartellverbot und Missbrauchsaufsicht perfekt funktionieren, wäre die Fusionskontrolle überflüssig, da jedes missbräuchliche Verhalten einfach ex post abgestellt werden könnte. Asymmetrische Informationen und eingeschränkte Rationalität verhindern jedoch in der Realität eine perfekte Missbrauchsaufsicht ebenso wie eine fehlerlose Durchsetzung des Kartellverbots, sodass durch die Fusionskontrolle bereits ex ante die Entstehung von Gefahrenlagen unterbunden werden soll, eben weil der Missbrauch von Marktmacht oder die Herausbildung kollusiven Verhaltens ex post nur schwer kontrollierbar ist. Institutionell stehen somit Fusionskontrolle einerseits sowie Kartellverbot und Missbrauchsaufsicht andererseits in einer gewissen substitutiven Beziehung: Umso besser Kartellverbot und Missbrauchsaufsicht funktionieren, desto weniger wichtig ist die Fusionskontrolle et vice versa. Sich dies zu vergegenwärtigen ist wichtig, weil die aktuelle GWB-Novelle sich fast ausschließlich auf die Erweiterung der Missbrauchsaufsicht fokussiert, die Fusionskontrolle jedoch nahezu unberührt lässt.

\section{Schärfung der Missbrauchsaufsicht für digitale Plattformen}

Auf Empfehlung der Monopolkommission wurden in Deutschland bereits 2017 mehrere Änderungen im Kartellrecht vorgenommen, um die Marktmacht digitaler Plattformen besser zu erfassen. ${ }^{24}$ In $\S 18$ Abs. 1 Nr. 3a GWB heißt es: „Insbesondere bei mehrseitigen Märkten und Netzwerken sind bei der Bewertung der Marktstellung eines Unternehmens auch zu berücksichtigen: (1) direkte und indi-

24 Vgl. Monopolkommission: Wettbewerbspolitik, a.a. O. 
rekte Netzwerkeffekte, (2) die parallele Nutzung mehrerer Dienste und der Wechselaufwand für die Nutzer, (3) seine Größenvorteile im Zusammenhang mit Netzwerkeffekten, (4) sein Zugang zu wettbewerbsrelevanten Daten, (5) innovationsgetriebener Wettbewerbsdruck." Diese Kriterien reflektieren die in der ökonomischen Literatur entwickelten Faktoren. ${ }^{25}$

$\S 18$ Abs. 3a GWB kann für das Bundeskartellamt und die zuständigen Gerichte bei der Beurteilung der Marktmacht einer Plattform eine Hilfestellung bieten, doch werden marktmächtigen Unternehmen dadurch weder zusätzliche Beschränkungen in Bezug auf ihr Verhalten auferlegt noch ändert sich die Schwelle, ab der ein Eingriff der Kartellbehörden erfolgen kann. Da aber Anreize zur Abschottung von Plattformmärkten durch Behinderung des Multi-Homing stark sind und der Wettbewerb schwieriger wieder zu beleben ist, sobald ein Markt gekippt ist, sollte die Erhaltung von Multi-Homing-Möglichkeiten ein zentrales Anliegen der Wettbewerbsbehörden sein. Gute Gründe sprechen dafür, in solchen Fällen bereits einzugreifen, bevor eine Plattform die Marktbeherrschung erlangt hat, da die Schäden für den Wettbewerb nahezu irreversibel oder zumindest schwer rückgängig zu machen sind. In Schweitzer et al. haben wir daher einen stufenweisen Ansatz für das Wettbewerbsrecht vorgeschlagen. ${ }^{26}$ Für Plattformen, die über relative oder überlegene Marktmacht verfügen, sollte die Beweislast auf die Plattform verlagert werden, wenn diese das Multi-Homing künstlich einschränken will, selbst wenn die betroffene Plattform (noch) nicht marktbeherrschend ist. Für die geplante 10. GWB-Novelle wird dieser Vorschlag nun in $\S 20$ Abs. 3a GWB-Entwurf (GWB-RefE) aufgegriffen, der laut Referentenentwurf wie folgt lauten soll: „Eine unbillige Behinderung im Sinne des Abs. 3 S. 1 liegt auch vor, wenn ein Unternehmen mit überlegener Marktmacht auf einem Markt im Sinne des $\S 18$ Abs. 3a die eigenständige Erzielung von positiven Netzwerkeffekten durch Wettbewerber behindert und hierdurch die ernstliche Gefahr begründet, dass der Leistungswettbewerb in nicht unerheblichem Maße eingeschränkt wird.“

Diese Regelung wird die Einschränkung von Multi-Homing bei Plattformen mit überlegener Marktmacht zwar nicht per se untersagen, aber die Beweislast umkehren. Ein Kippen des Marktes wird zwar letztlich nicht immer verhindert. Wenn etwa Unternehmen nachweisen können, dass Multi-Homing Ineffizienzen generiert und es daher sachlich gerechtfertigt ist, dies einzuschränken, wird der Markt weiterhin in ein Monopol umkippen. Jedoch wird eine Klausel, welche die Beweislast auf Plattformen mit überlegener Marktmacht überträgt, zumindest einen gewissen Rück-

25 Vgl. D. S. Evans, R. Schmalensee: The Antitrust Analysis ..., a.a. O. 26 Vgl. H. Schweitzer, J. Haucap, W. Kerber, R. Welker, a. a. O. halt gegen das Kippen bieten und dazu beitragen, den Wettbewerb zu erhalten, wenn Multi-Homing möglich ist. Dies ist sinnvoll, weil bei Multi-Homing Wettbewerb zwischen den Plattformen nicht automatisch einen Verlust von Netzwerkeffekten bedeutet. Es ist daher sinnvoll, den Kartellbehörden bessere Möglichkeiten einzuräumen, das Erschweren von Multi-Homing schon frühzeitig zu unterbinden.

Weiter wird die Missbrauchsaufsicht gestärkt, indem das von uns in Schweitzer et al. entwickelte Konzept der Intermediationsmacht dem Referentenentwurf zufolge als neuer $\S 18$ Abs. 3b in das GWB aufgenommen werden soll: „Bei der Bewertung der Marktstellung eines Unternehmens, das als Vermittler auf mehrseitigen Märkten tätig ist, ist insbesondere auch die Bedeutung der von ihm erbrachten Vermittlungsdienstleistungen für den Zugang zu Beschaffungs- und Absatzmärkten zu berücksichtigen.“ Mit der Übernahme des Konzepts der Intermediationsmacht beschreitet das deutsche Kartellrecht auch international Neuland.

Die am weitesten gehende Schärfung der Missbrauchsaufsicht besteht jedoch in der geplanten Einführung des neuen § 19a GWB-RefE, der eine regulierungsähnliche Missbrauchsaufsicht für Plattformen einführen soll. Konkret soll das Bundeskartellamt durch Verfügung feststellen können, dass einem Unternehmen, das in erheblichem Umfang auf Plattformmärkten tätig ist, eine „überragende marktübergreifende Bedeutung“ (ÜMÜB) für den Wettbewerb zukommt. Das Bundeskartellamt kann einer solchen ÜMÜB-Plattform untersagen, „1. beim Vermitteln des Zugangs zu Beschaffungs- und Absatzmärkten die Angebote von Wettbewerbern anders zu behandeln als eigene Angebote; 2. Wettbewerber auf einem Markt, auf dem das betreffende Unternehmen seine Stellung auch ohne marktbeherrschend zu sein schnell ausbauen kann, unmittelbar oder mittelbar zu behindern, sofern die Behinderung geeignet wäre, den Wettbewerbsprozess erheblich zu beeinträchtigen; 3. durch die Nutzung der auf einem beherrschten Markt von der Marktgegenseite gesammelten wettbewerbsrelevanten Daten, auch in Kombination mit weiteren wettbewerbsrelevanten Daten aus Quellen außerhalb des beherrschten Marktes, auf einem anderen Markt Marktzutrittsschranken zu errichten oder zu erhöhen oder andere Unternehmen in sonstiger Weise zu behindern oder Geschäftsbedingungen zu fordern, die eine solche Nutzung zulassen; 4. die Interoperabilität von Produkten oder Leistungen oder die Portabilität von Daten zu erschweren und damit den Wettbewerb zu behindern; 5. andere Unternehmen unzureichend über den Umfang, die Qualität oder den Erfolg der erbrachten oder beauftragten Leistung zu informieren oder innen in anderer Weise eine Beurteilung des Wertes dieser Leistung zu erschweren.“ 
Damit kann das Bundeskartellamt den ÜMÜB-Plattformen ex ante bestimmte Verhaltensweisen, wie etwa die Selbstbevorzugung, die Nutzung bestimmter Daten oder auch das Ausnutzen von Informationsasymmetrien, untersagen. Durch diese neue Vorschrift wird die kartellrechtliche Missbrauchsaufsicht deutlich geschärft und auch regulierungsähnlicher. Die Vielzahl unbestimmter Rechtsbegriffe sowie die Gefahr, dass die strikte Untersagung bestimmter Praktiken Effizienzgewinne und Wettbewerbsvorstöße verhindern können, haben jedoch gerade in Bezug auf den geplanten § 19a GWB zu sehr kritischen Einschätzungen geführt. $^{27}$

Weitere Stärkungen soll die Missbrauchsaufsicht zudem erstens durch die Streichung des Begriffs „kleiner und mittlerer" vor Unternehmen als Voraussetzung für einen Missbrauch bei relativer Marktmacht (§ 20 Abs. 1 GWB) erfahren sowie zweitens durch Regelungen zum Datenzugangsanspruch. Diese Streichung trägt der Erkenntnis Rechnung, dass nicht nur der Mittelstand vor marktmächtigen Digitalkonzernen geschützt werden muss und eine Abhängigkeit auch bei großen Unternehmen bestehen kann. Zusammenfassend lassen sich also im Bereich der Missbrauchskontrolle zahlreiche Verschärfungen feststellen, sodass das sogenannte GWB-Digitalisierungsgesetz in der Tat als „großer Wurf" bezeichnet werden kann.

\section{Fusionskontrolle: Killerakquisitionen und die „Kill Zone“}

Bis zum Inkrafttreten der 9. GWB-Novelle $2017^{28}$ bestand ein Manko der Fusionskontrolle darin, dass eine Reihe von Fusionen nicht kartellrechtlich geprüft werden konnten, da sich diese unterhalb der sogenannten Aufgreifschwellen bewegten, die wiederum allein auf den Umsätzen der Unternehmen beruhten. Bei stark wachsenden Plattformen mit unentgeltlichen Leistungen auf einer Marktseite kann dies jedoch dazu führen, dass wirtschaftlich sehr bedeutsame Fusionen kartellrechtlich ungeprüft bleiben. Um dies zu beheben, wurde 2017 in Deutschland und Österreich eine neue transaktionswertbasierte Aufgreifschwelle in die Fusionskontrolle eingeführt.

Ein weiteres Anliegen vieler Wettbewerbsexperten sind sogenannte „Killerakquisitionen“29. Eigentlich bezieht sich der Begriff auf Übernahmen in der Pharmaindustrie. Dort ist zu beobachten, dass nicht selten Unternehmen auch deshalb einen Konkurrenten übernehmen, um dessen Forschung

27 Vgl. etwa Monopolkommission: 10. GWB-Novelle - Herausforderungen auf digitalen und regionalen Märkten begegnen!, Policy Brief, Nr. 4, Januar 2020.

28 Vgl. dazu C. Kersting, R. Podszun (Hrsg.): Die 9. GWB-Novelle, München 2017.

29 Vgl. C. Cunningham, F. Ederer, S. Ma: Killer Acquisitions, Working Paper, 2019, https://ssrn.com/abstract=3241707 (19.3.2020). einzustellen, sofern diese ein Patent des übernehmenden Unternehmens bedroht. Übertragen auf die digitale Wirtschaft ist mit einer Killerakquisition gemeint, dass große Plattformen wie die GAFAM-Unternehmen (potenzielle) Wettbewerber erwerben, um den potenziellen Wettbewerb abzuwürgen. Die Übernahme von WhatsApp durch Facebook ist ein oft genanntes Beispiel für eine solche potenzielle „Killerakquisition“, da so die etwaigen Ambitionen von WhatsApp, ebenfalls ein soziales Netzwerk zu werden, das dann mit Facebook konkurriert hätte, zerstört wurden.

Bei vielen der „konglomeraten Fusionen“ in der Digitalwirtschaft sind der Erwerber und das Ziel jedoch in unterschiedlichen Märkten tätig, da sie unterschiedliche Dienstleistungen mit unterschiedlichen Funktionalitäten anbieten, die unterschiedliche Bedürfnisse befriedigen können. Wenn Erwerber und Zielunternehmen auf verschiedenen Märkten tätig sind, werden diese konglomeraten Fusionen daher oft nicht von den Wettbewerbsbehörden unterbunden, weil sie nicht einem gemeinsamen Markt, sondern getrennten Märkten zugerechnet werden.

Um die Fusionskontrolle zu stärken, werden nun verschiedene Vorschläge diskutiert: Ein Vorschlag besteht darin, Märkte weiter zu definieren, etwa als allgemeine „Märkte für Aufmerksamkeit" ${ }^{30}$. Da die Aufmerksamkeit der Menschen begrenzt ist, konkurrieren Firmen wie Netflix, Facebook, YouTube und viele andere um die Aufmerksamkeit der Nutzer, entweder um direkt Einnahmen zu generieren (wie Netflix) oder um indirekt Einnahmen durch mehr Werbung zu generieren (wie bei YouTube und Facebook). Ein Ansatz, der die Marktabgrenzungen erweitert, hat jedoch den Nachteil, dass - während Facebook und WhatsApp und viele andere Dienste in einem „Markt für Aufmerksamkeit" zwar besser in der Fusionskontrolle adressiert werden können - eine solch breite Marktdefinition am Ende die kartellrechtliche Missbrauchsaufsicht gefährden kann, da weniger Unternehmen auf einem solchen Markt marktbeherrschend sein würden.

Ein zweiter Vorschlag sieht vor, dass Wettbewerbsbehörden im Rahmen der Fusionskontrolle auch die Erwerbsstrategien von Unternehmen berücksichtigen können. Während die Fusionskontrolle traditionell jede Übernahme als einen separaten Fall betrachtet, erfordern die Akquisitionsstrategien marktbeherrschender Unternehmen auch eine Analyse des Gesamtbildes, damit die Behörden die Wettbewerbsfolgen mehrerer Übernahmen in Summe bewerten können. Nachteilig an einem solchen Ansatz ist sicherlich die zunehmende Rechtsunsicherheit, die mit solch neuartigen Vorgehensweisen verbunden wäre.

30 A. Prat, T. Valletti: Attention Oligopoly, Working Paper, 2019, https:// papers.ssrn.com/sol3/papers.cfm?abstract_id=3197930 (19.3.2020). 
Ganz generell kann jedoch eine Ausweitung der Fusionskontrolle das Problem der sogenannten „Kill Zone“ verschärfen, das vom Problem der Killerakquisitionen zu unterscheiden ist. Mit der „Kill Zone“ ist gemeint, dass Start-ups, die mögliche Konkurrenzprodukte zu den GAFAM-Plattformen entwickeln wollen, womöglich schon deswegen erst gar keine Finanzierung erhalten, weil etwaige Kapitalgeber befürchten, dass die GAFAM-Plattformen sehr aggressiv gegen diese potenzielle Konkurrenz vorgehen werden, sofern sie diese nicht übernehmen können. Anders ausgedrückt haben Start-ups womöglich weniger Möglichkeiten eine Finanzierung zu erhalten, wenn sie nicht als Exit-Option die Möglichkeit haben, an die GAFAM-Plattformen zu verkaufen. ${ }^{31}$

Das „Kill Zone“-Problem kann nun gerade nicht durch eine Ausweitung und Schärfung der Fusionskontrolle gelöst werden, da die Befürchtung der Investoren meist gerade nicht in einer Übernahme durch die GAFAM-Plattformen besteht (dies scheint sogar ein Exit-Plan von Start-ups zu sein), sondern die aggressive Konkurrenz durch die GAFAM-Plattformen befürchtet wird, wenn das Start-up nicht übernommen werden kann. Bei retrospektiv als besonders kritisch eingestuften Akquisitionen wie Googles Übernahmen von DoubleClick im Jahr 2007 und Waze im Jahr 2013 sowie Facebooks Kauf von Instagram im Jahr 2012 und von WhatsApp im Jahr 2014 oder Microsofts Akquisition von Skype im Jahr 2011 stellt sich stets die Frage nach der kontrafaktischen Situation, die kaum zu bestimmen ist. Möglicherweise hätten sich aus den übernommenen Unternehmen potente Wettbewerber entwickelt. Womöglich hätten die GAFAM-Plattformen durch aggressiven Wettbewerb aber auch ein Wachstum der Konkurrenz unterbunden oder die betroffenen Unternehmen wären ohne den Push durch die GAFAM-Plattformen überhaupt nie in diesem Maße erfolgreich geworden. Dies ex post zu evaluieren ist bereits schwierig, es ex ante zu prognostizieren für eine Behörde vermutlich in vielen Fällen fast unmöglich.

Wie also sollte man mit den vermeintlichen Killerakquisitionen in der Digitalwirtschaft umgehen? Diskutiert werden sehr weitgehende Vorschläge, wie die Umkehr der Beweislast für potenzielle Killerakquisitionen oder für „Unternehmen mit überragender marktübergreifender Bedeutung für den Wettbewerb“ nach § 19a GWB-RefE. Allerdings ist der Beweis, dass eine Fusion den Wettbewerb nicht schwächt, für einzelne Unternehmen kaum zu erbringen, da diese anders als die Kartellbehörden - nicht auf die Daten ihrer

31 Siehe dazu Antitrust-Konferenz des Stigler Centers 2018: A. Schechter: Google and Facebook's "Kill Zone": "We've Taken the Focus Off of Rewarding Genius and Innovation to Rewarding Capital and Scale", Pro Market Blog, 25.5.2018, https://promarket.org/google-facebooks-kill-zone-weve-taken-focus-off-rewarding-genius-innovationrewarding-capital-scale/ (19.3.2020).
Konkurrenz zurückgreifen können. Alternativ wird auch die Ex-post-Kontrolle von Fusionen als Teil der Missbrauchsaufsicht erörtert. Auch dies schafft nicht unerhebliche Schwierigkeiten, wenn das Unternehmen und die Mitarbeiter bereits in ein anderes Unternehmen integriert wurden. Die geplante GWB-Novelle setzt daher nun vor allem auf die Schärfung der allgemeinen Missbrauchsaufsicht. Vor dem Hintergrund des teils substitutiven Verhältnisses erscheint eine solche Vorgehensweise durchaus ratsam.

\section{Rolle von Daten in der digitalen Ökonomie}

Neben der zunehmenden Bedeutung von Plattformen als Intermediationsform ist das zweite Merkmal der digitalen Wirtschaft die umfangreiche Nutzung von Daten, um die Effizienz bei Produktdesign, Fertigung, Vertrieb, Marketing und nahezu allen Teilen der Wertschöpfungskette zu steigern. Aus wettbewerbspolitischer Sicht stellt sich die Frage, ob der „Datenschatz“ eines Unternehmens die Quelle eines solchen Wettbewerbsvorteils sein kann, der es sehr unwahrscheinlich oder gar unmöglich werden lässt, dass andere Unternehmen in absehbarer Zukunft aufholen können. Anders ausgedrückt stellt sich die Frage, ob der Zugang zu einigen Daten so bedeutsam sein kann, dass der Wettbewerbsvorteil eines Unternehmens von keinem Wettbewerber realistischerweise mehr erreicht werden kann. ${ }^{32}$ Aus verbraucherpolitischer Sicht ergeben sich zumindest bei personenbezogenen Daten neue Herausforderungen beim Schutz der Privatsphäre. Intensiv diskutiert wird aktuell die Frage, unter welchen Bedingungen Dritten der Zugang zu Daten gewährt werden sollte und ob er ihnen auch unterhalb der für sogenannte „wesentliche Einrichtungen“ entwickelten gesetzlichen Schwelle gewährt werden soll oder, anders ausgedrückt, ob der bisherige Zugang Dritter im Vergleich zum Zugang zu klassischen wesentlichen Einrichtungen erleichtert werden sollte.

Die Grundidee, dass marktbeherrschende Unternehmen möglicherweise Dritten Zugang zu ihrem physischen und geistigen Eigentum gewähren müssen, ist nicht ganz neu. In vielen Infrastruktur- oder Versorgungsbranchen wie Telekommunikation, Elektrizität, Gas, Eisenbahnen und Post wurde der Wettbewerb dadurch ermöglicht, dass etablierte Unternehmen verpflichtet wurden, ihre Netze zu öffnen und Dritten Zugang zu ihren Einrichtungen zu gewähren. ${ }^{33}$ Die rechtliche Schwelle, die überschritten werden muss, bevor Dritten der Zugang durch Regulierung oder Wettbewerbsrecht vorgeschrieben ist, ist jedoch traditionell recht

32 Vgl. A. Argenton, J. Prüfer: Search Engine Competition with Network Externalities, in: Journal of Competition Law \& Economics, 8. Jg. (2012), H. 1, S. 73-105; I. Graef: EU Competition Law, Data Protection and Online Platforms: Data as Essential Facility, Alphen aan den Rijn 2016.

33 Vgl. Monopolkommission: 13. Hauptgutachten: Wettbewerbspolitik in Netzstrukturen, Baden-Baden 2000. 
hoch. Umfangreiche rechtliche Tests mussten traditionell bestanden werden, bevor den Infrastrukturbesitzern Zugangsverpflichtungen auferlegt wurden. Die (ökonomischen) Gründe, den Zugang Dritter nicht zu leicht zu gewähren, waren zweifach: Erstens leiden Investitionsanreize für den Bau und die Instandhaltung teurer Anlagen, wenn auch Dritte Zugang zu den Anlagen haben, ohne die Investitionsrisiken zu teilen. Und zweitens war die Nutzung dieser Einrichtungen oft rivalisierend. Übernimmt ein Telekommunikationsbetreiber eine Kundenleitung (die letzte Meile) von einem anderen Betreiber (z. B. über die Entbündelung des Teilnehmeranschlusses), kann dieser diese Leitung nicht mehr nutzen. Auch dort, wo die Kapazitäten der Eisenbahn begrenzt sind, ist die Nutzung der Infrastruktur oft rivalisierend.

Im Gegensatz dazu kann dies bei Daten ganz anders sein, und die negativen Auswirkungen einer Zugangsverpflichtung auf Investitionsanreize können geringer sein. Erstens sind Daten nicht rivalisierend in der Nutzung. Selbst wenn ein Dritter bestimmte Daten verwendet, kann der ursprüngliche Dateninhaber die Daten nach wie vor verwenden. Daten werden nicht ver-, sondern gebraucht. Diese Logik impliziert als Faustregel, dass ein Zugangsanspruch grundsätzlich ab einem niedrigeren Schwellenwert möglich sein sollte als der Zugang zu traditionellen infrastrukturbasierten wesentlichen Einrichtungen, die oft einen gewissen Grad an Rivalität aufweisen und typischerweise mit erheblichen Investitionen verbunden sind. Und zweitens können Daten teilweise auch als Nebenprodukt eines anderen Dienstes ohne hohe Investitionskosten anfallen, aber dennoch schwer zu duplizieren sein, z. B. in Energienetzen, Verkehrsdaten oder bei maschinell erzeugten Daten. Natürlich verursacht die Erhebung von Daten teilweise auch erhebliche Kosten, aber dies ist nicht immer der Fall. Somit sollte die gesetzliche Schwelle für einen Zugangsanspruch Dritter bei Daten tendenziell niedriger sein als es die Schwelle bei klassischen wesentlichen Einrichtungen ist. Gleichwohl gibt es auch zusätzliche Möglichkeiten der Rechtfertigung einer Zugangsverweigerung: Datenschutz. Bei personenbezogenen Daten verhindern Datenschutzbestimmungen in der Regel schon den Zugang für Dritte. Für anonymisierte Daten oder nicht personenbezogene Daten könnte der Datenzugang jedoch leichter gewährt werden, um den Wettbewerb zu fördern. Bei personenbezogenen Daten hilft die in der Datenschutzgrundverordnung garantierte Portabilität, den Wettbewerb zu fördern.

Ein Datenzugang für Dritte ist insbesondere dann von Bedeutung, wenn ein Unternehmen bereits auf einem Markt marktbeherrschend ist oder wenn Unternehmen von anderen abhängig sind, die über relative oder überlegene Marktmacht verfügen. Darüber hinaus sollten freiwillige Vereinbarungen zum Datenaustausch zwischen Unter- nehmen mit weniger Argwohn betrachtet werden, als dies bisher im Kartellrecht der Fall war. In der geplanten GWBNovelle soll ein Anspruch auf Datenzugang zum einen in $\S 19$ Abs. 2 Nr. 4 GWB geschaffen werden, sodass Daten als wesentliche Einrichtung (essential facility) begriffen werden können. Zum anderen wird neu § 20 Abs. 1a GWB eingeführt, demzufolge sich eine Abhängigkeit auch daraus ergeben kann, „dass ein Unternehmen für die eigene Tätigkeit auf den Zugang zu Daten angewiesen ist, die von einem anderen Unternehmen kontrolliert werden. Die Verweigerung des Zugangs zu solchen Daten kann auch dann eine unbillige Behinderung darstellen, wenn ein Geschäftsverkehr für diese Daten bislang nicht eröffnet ist." Da $\S 20$ Abs. 1a GWB noch über $\S 19$ Abs. 2 Nr. 4 GWB in dem Sinne hinausgeht, dass die Schwelle für die Feststellung eines Missbrauchs deutlich niedriger ist, könnte $§ 19$ Abs. 2 Nr. 4 GWB im Grunde überflüssig sein. Das Absenken der Eingriffsschwelle ist ökonomisch jedoch gut zu begründen.

\section{Plattformen und Informationsverzerrungen}

Da Verbraucher in der digitalen Ökonomie zunehmend auf Informationsintermediäre angewiesen sind, kann es vorkommen, dass einige Intermediäre hinsichtlich der Qualität der Informationsvermittlung keiner effektiven wettbewerblichen Disziplinierung unterliegen, selbst wenn den Verbrauchern grundsätzlich Ausweichmöglichkeiten zur Verfügung stehen. ${ }^{34}$ Ein Indiz dafür ist es, wenn Verbraucher auf eine verzerrte Informationsvermittlung - etwa durch Verwendung von Rankingkriterien, die nicht auf Nutzerpräferenzen basieren - nicht in einem Maße mittels Abwanderung reagieren, dass die aus der Informationsverzerrung erwarteten Vorteile kleiner sind als die durch Abwanderungen erwarteten Einbußen. Die Gründe dafür können vielfältig sein. Informationen wie auch Rankings und Bewertungen haben aus Nutzersicht oft die Eigenschaft von Vertrauensgütern. Entsprechend der Funktion von Informationsintermediären, die Suchkosten zu senken, fehlt es den Nutzern an einem hinreichenden Anlass und/oder an Möglichkeiten, die Qualität der innen vermittelten Information zu überprüfen. Aus dem Vertrauen der Nutzer folgt ein Verhaltensspielraum des Intermediärs, den dieser zu einer Steuerung des Verhaltens der Verbraucher gemäß seinen wirtschaftlichen Eigeninteressen nutzen kann. So kann eine Plattform z.B. eigene Produkte oder Dienstleistungen oder solche mit besonders hohen Provisionszahlungen in der Trefferliste - ob auf einer allgemeinen Suchmaschine, einer Vergleichsplattform oder einer Handelsplattform priorisieren, ohne dass viele Nutzer dies bemerken. Betreiben Verbraucher kein systematisches Multi-Homing, so werden sie sich oft durch die Reihenfolge der Treffer leiten

34 Der folgende Abschnitt basiert auf unseren Ausführungen in $\mathrm{H}$. Schweitzer, J. Haucap, W. Kerber, R. Welker, a. a. O. 
lassen. Je größer die Zahl der Nutzer der Plattform, die sich auf die Informationsbereitstellung der Plattform verlässt, desto größer kann der Grad der Wettbewerbsverfälschung auf angrenzenden Märkten sein.

Eine solche nutzerseitig unerkannte und daher wettbewerblich nicht disziplinierte Ausnutzung von Informationsasymmetrien durch Informationsintermediäre kann auch deutlich unterhalb einer Marktbeherrschung zu Fehlsteuerungen auf sonst wettbewerblichen Märkten führen, denn aus ökonomischer Sicht induziert eine Informationsasymmetrie immer einen gewissen Grad an Marktmacht im Vergleich zum hypothetischen Ideal vollständigen Wettbewerbs. Das Ausmaß der wettbewerblich nicht kontrollierten Spielräume eines Anbieters hängt dabei primär vom Ausmaß der Informationskosten ab. Je höher die Informationskosten der Nachfrager sind, desto ausgeprägter sind tendenziell die Verhaltensspielräume der Anbieter.

Zwischen der Marktstruktur und den durch Informationsasymmetrien ermöglichten Verhaltensspielräumen bestehen nicht unbedingt lineare Zusammenhänge. Je einfacher und günstiger es für die Nachfrager ist, eine zweite Meinung einzuholen, desto eher können Informationsasymmetrien reduziert werden. Das Vergleichen oder Einholen zweiter Meinungen wird dabei umso einfacher sein, je mehr Anbieter derselben Leistung es auf dem Markt gibt. Im Extremfall des Monopols hingegen ist ein Vergleich oder das Einholen der zweiten Meinung per Definition ausgeschlossen, somit eine Informationsasymmetrie besonders schwer durch das Vergleichen von alternativen Anbietern zu beseitigen. $\mathrm{Zu}$ bedenken ist jedoch auch, dass große Anbieter tendenziell einer stärkeren Kontrolle durch Medien und Öffentlichkeit unterliegen als viele kleine Anbieter. Möglicherweise kann ab einem gewissen Konzentrationsmaß eine weitere Abnahme der Anbieterzahl zu einem Anstieg der Informationskosten für Nachfrager, aufgrund eines weiteren Rückgangs der Vergleichsmöglichkeiten, führen und damit zu einer Stärkung der Informationsasymmetrien und einer Zunahme der wettbewerblich nicht kontrollierten Verhaltensspielräume. Zur Gewährleistung eines Schutzes der
Funktionsfähigkeit von Märkten, in denen Informationsintermediäre eine erhebliche Rolle spielen, ist es daher ratsam, jenseits des Kartellrechts zusätzliche Verbraucherschutzmaßnahmen zu ergreifen, um ein Ausnutzen von Informationsasymmetrien durch Intermediationsintermediäre möglichst zu unterbinden.

\section{Fazit zu den neuen Wettbewerbsregeln}

Während digitale Plattformen den Wettbewerb auf vielen Märkten intensiviert haben, besteht aufgrund des kippligen „winner takes all“-Charakters dieser Märkte auch ein erhöhtes Risiko einer Marktabschottung durch große Plattformen. Daher gibt es gute Gründe für eine Nachschärfung des Kartellrechts, wie sie jetzt im Rahmen der 10. GWB-Novelle geplant ist. Dass dabei der Fokus der Kartellrechtsnovelle auf der Ausweitung der Missbrauchsaufsicht liegt und nicht auf der Fusionskontrolle, lässt sich ökonomisch gut begründen, da eine schärfere und besser greifende Missbrauchsaufsicht, die wettbewerbswidriges Verhalten von Unternehmen wirksam adressiert, die parallele Ausweitung der Fusionskontrolle weniger bedeutsam macht. Eine Ausweitung der Fusionskontrolle ist gerade schwierig, weil sich dadurch zwar das Problem der sogenannten Killerakquisitionen besser adressieren ließe, das nicht weniger relevante Problem der GAFAM-,,Kill Zone“ aber noch verschärft würde. Zugleich ist die Prognose von Wettbewerbswirkungen ex ante (also in der Fusionskontrolle) deutlich schwieriger als die Bewertung von Unternehmensverhalten ex post (wie in der Missbrauchsaufsicht). Es erscheint daher durchaus klug, das Thema Fusionskontrolle erst in der 11. GWB-Novelle zu adressieren. Darüber hinaus gilt es auch, horizontale Vereinbarungen (etwa zum Datentausch) neu zu bewerten, wie es etwa die Expertenkommission Wettbewerbsrecht 4.0 empfohlen hat, ${ }^{35}$ ebenso wie vertikale Beschränkungen des Internethandels. Es ist daher zu begrüßen, dass die EU-Kommission auch in diesen Bereichen die Leitlinien überarbeiten will.

35 Expertenkommission Wettbewerbsrecht 4.0, a. a. O

Title: The Platform Economy: New Competition Rules - Renaissance of Antitrust Control of the Abuse of Market Power Abstract: While digital platforms have intensified competition in many markets, there is also a higher risk of market foreclosure due to the "winner takes all"-nature of these markets. Hence, there are good reasons for a strengthening of competition law, as now planned with Germany's competition law reform. Focussing on the control of abuse of market power instead of merger control is well founded from an economic perspective, as a stricter control of abusive behaviour makes merger control less important. Strengthening merger control is difficult: While this could better address the problem of so-called killer acquisitions, it would also worsen the problem of GAFAM kill zones, which is equally relevant.

JEL Classification: K21, K23, L40, L86 